\title{
Gravitational Uncertainty and Black Hole Remnants
}

\author{
Kourosh Nozari* $^{*}$ and S. Hamid Mehdipour ${ }^{\dagger}$ \\ Department of Physics, Faculty of Basic Sciences, University of Mazandaran, \\ P. O. Box 47416-1467, Babolsar, IRAN
}

October 2005

\begin{abstract}
Possible existence of black holes remnants provides a suitable candidates for dark matter. In this paper we study the possibility of existence for such remnants. We consider quantum gravitational induced corrections of black hole's entropy and temperature to investigate the possibility of such relics. Observational scheme for detection of these remnants and their cosmological constraints are discussed.
\end{abstract}

PACS: 04.60.-m , 04.70.-s, 04.70.Dy, 95.35.+d

Key Words: Quantum Gravity, Generalized Uncertainty Principle, Varying Speed of Light Models, Black Holes Thermodynamics, Dark Matter

\footnotetext{
*knozari@umz.ac.ir

†h.mehdipour@umz.ac.ir
} 


\section{Introduction}

It is by now widely accepted that dark matter (DM) constitutes a substantial fraction of the present critical energy density in the Universe. However, the nature of DM remains an open problem. There exist many DM candidates, most of them are non-baryonic weakly interacting massive particles (WIMPs), or WIMP-like particles [1]. By far the DM candidates that have been more intensively studied are the lightest supersymmetric (SUSY) particles such as neutralinos or gravitinos, and the axions (as well as the axinos). There are additional particle physics inspired dark matter candidates [1]. A candidate which is not as closely related to particle physics is the relics of primordial black holes (Micro Black Holes) [2,3]. Certain inflation models naturally induce a large number of such a black holes. As a specific example, hybrid inflation can in principle yield the necessary abundance of primordial black hole remnants for them to be the primary source of dark matter $[4,5]$. In recent years it has been suggested that measurements in quantum gravity should be governed by generalized uncertainty principle (GUP). In fact, some evidences from string theory, quantum geometry and black hole physics, have led some authors to re-examine usual uncertainty principle of Heisenberg [6-13]. These evidences have origin on the quantum fluctuations of the background space-time metric. Existence of a minimal length scale on the order of Planck length is an immediate consequence of GUP. Introduction of this idea has attract considerable attention and many authors considered various problems in the framework of generalized uncertainty principle [1428]. The issue of black holes remnants has been considered by some authors. Adler and his coworkers have argued that contrary to standard viewpoint, GUP may prevent small black holes total evaporation in exactly the same manner that the uncertainty principle prevents the Hydrogen atom from total collapse [29]. Chen considering inflation induced primordial black holes, have investigated the issue of stability of such relics [30]. Recently, Varying Speed of Light (VSL), as a new conjecture, which has been proposed to solve the problems of standard cosmology, has attract some attentions. After introduction of this conjecture, several alternative VSL theories have been proposed and some of their novel implications have been examined extensively [31-35].

In this paper the issue of black hole remnants will be considered in the framework of both GUP and VSL. The main consequence of this combination is related to the stability

problem of possible remnants. In forthcoming sections first we show that GUP provides a reasonable framework for VSL. Then using a simple VSL model we will show that if one 
consider both the effect of GUP and VSL on the thermodynamics of black holes, the results of Bekenstein-Hawking concerning total evaporation of black holes should be re-examined. In Bekenstein-Hawking approach the total evaporation of micro black hole is possible. Here we will see that it is possible to have relics of evaporating black holes which can be considered as a possible candidate for dark matter. The structure of the paper is as follows: in section 2 we show that VSL can be considered as an immediate consequence of GUP. Section 3 is devoted to the quantum gravitational corrected black hole thermodynamics. Some numerical calculations have been down and their physical results are discussed. The paper follows by conclusions and discussion regarding observational scheme for detection of such relics and their cosmological constraints in section 4 .

\section{Preliminaries}

As has been revealed in introduction, usual uncertainty principle of quantum mechanics, the so-called Heisenberg uncertainty principle, should be re-formulated due to noncommutative nature of spacetime at Planck scale. As a consequence, it has been indicated that in quantum gravity there exists a minimal observable distance on the order of the Planck length which governs on all measurements in extreme quantum gravity limit. In the context of string theories, this observable distance is referred to GUP. A generalized uncertainty principle can be formulated as

$$
\Delta x \geq \frac{\hbar}{\Delta p}+\text { const.G } \Delta p,
$$

which, using the minimal nature of $l_{P}$ can be written as,

$$
\Delta x \geq \frac{\hbar}{\Delta p}+\frac{\alpha^{\prime} l_{p}^{2} \Delta p}{\hbar} .
$$

The main consequence of GUP is that measurement of the position is possible only up to Planck length, $l_{P}$. So one can not setup a measurement to find more accurate particle position than Planck length, and this means that the notion of locality breaks down. It is important to note that there are more generalization which contain further terms in right hand side of equation (2) (see [36]), but in some sense regarding dynamics, equation (2) has more powerful physical grounds. Suppose that

$$
\Delta x \sim x, \quad \Delta p \sim p, \quad p=\hbar k, \quad x=\bar{\lambda}=\frac{\lambda}{2 \pi} .
$$


Therefore one can write,

$$
\bar{\lambda}=\frac{1}{k}+\alpha^{\prime} l_{p}^{2} k \quad \text { and } \quad \omega=\frac{c}{\bar{\lambda}} .
$$

In this situation the dispersion relation becomes,

$$
\omega=\omega(k)=\frac{k c}{1+\alpha^{\prime} l_{p}^{2} k^{2}} .
$$

This relation can be described in another viewpoint. By expansion of $\left(1+\alpha^{\prime} l_{p}^{2} k^{2}\right)^{-1}$ and neglecting second and higher order terms of $\alpha^{\prime}$, we find that $\omega=k c\left(1-\alpha^{\prime} l_{p}^{2} k^{2}\right)$. This can be considered as $\omega=k c^{\prime}$ where $c^{\prime}=c\left(1-\alpha^{\prime} l_{p}^{2} k^{2}\right)$. This relation indicates the possibility of variation in $c$. Accepting the possibility of variation in $c$, one can consider its time variation also. So we consider the time dependence of light speed.

Actually, there are some evidences indicating that fine structure constant, $\alpha=\frac{e^{2}}{\hbar c}$ is not constant [11]. The question then arises that which of the quantities: $e, c$ or $\hbar$ are variable? A possible situation is the variation in $c$. This is referred as varying speed of light (VSL) theories in literatures. After introduction of this idea several varying speed of light models have been proposed to solve the problems of standard cosmology. One of the simplest of these models is the model proposed by Barrow [35]. Barrow has considered the speed of light as

$$
c(t)=c_{0} a^{n}(t)
$$

where $c_{0}$ and $n$ are constant. Using this form of $c(t)$ to solve problems of standard cosmology, some constraint will be imposed on the value of $n$, depending on the nature of the problems. For example if we consider the equation of state for matter content of the Universe as $p=(\gamma-1) \rho c^{2}(t)$, then exact solutions with varying $c(t)$ and $G(t)$, restrict $n$ to the following limit

$$
\begin{aligned}
& n \leq-1 \text { for } \gamma=4 / 3 \quad \text { Radiation Dominated Era } \\
& n \leq-1 / 2 \text { for } \gamma=1
\end{aligned}
$$

\section{Black Holes Thermodynamics}

In the current standard viewpoint, small black holes emit black body radiation at the Hawking temperature,

$$
T_{H} \approx \frac{\hbar c^{3}}{8 \pi G M}=\frac{M_{P}^{2} c^{2}}{8 \pi M},
$$


where $M_{P}=\sqrt{\frac{\hbar c}{G}}$ is the Planck mass and we have set $k_{B}=1$. The related entropy is obtained by integration of $d S=c^{2} T^{-1} d M$ which is the standard Bekenstein entropy,

$$
S_{B}=\frac{4 \pi G M^{2}}{\hbar c}=4 \pi \frac{M^{2}}{M_{P}^{2}} \text {. }
$$

If one consider the GUP as equation (2), the last two equations become respectively,

$$
T_{G U P}=\frac{M c^{2}}{4 \pi}\left[1 \mp \sqrt{1-\frac{M_{P}^{2}}{M^{2}}}\right],
$$

and

$$
S_{G U P}=2 \pi\left[\frac{M^{2}}{M_{P}^{2}}\left(1-\frac{M_{P}^{2}}{M^{2}}+\sqrt{1-\frac{M_{P}^{2}}{M^{2}}}\right)-\ln \left(\frac{M+\sqrt{M^{2}-M_{P}^{2}}}{M_{P}}\right)\right] .
$$

In equation (10), to recover the corresponding result in the limit of large mass $\left(T_{H}\right)$, one should consider the minus sign. These equations strongly suggest the existence of black holes remnants. As it is evident from figure 2, in the framework of GUP black hole can evaporate until when it reachs the Planck mass. In this view point black hole remnants are stable. Now consider the case of VSL. For simplicity we assume that only $c$ is varying and $G$ and $\hbar$ are constant which we set $G=\hbar=1$. In this situation $M_{P}=\sqrt{c(t)}$. This is a novel concept: a time-varying Planck mass!. It means that Planck scales are varying with time and are actually cosmological models dependent via dependence of $c(t)$ to cosmological scale factor. The corresponding equations both in Hawking-Bekenstein and GUP viewpoint will become as follows respectively,

$$
\begin{gathered}
T_{H}^{(V S L)}(t)=\frac{c^{3}(t)}{8 \pi M}, \\
S_{B}^{(V S L)}(t)=\frac{4 \pi M^{2}}{c(t)}, \\
T_{G U P}^{(V S L)}(t)=\frac{M c^{2}(t)}{4 \pi}\left[1-\sqrt{1-\frac{c(t)}{M^{2}}}\right],
\end{gathered}
$$

and

$$
S_{G U P}^{(V S L)}(t)=2 \pi\left[\frac{M^{2}}{c(t)}\left(1-\frac{c(t)}{M^{2}}+\sqrt{1-\frac{c(t)}{M^{2}}}\right)-\ln \left(\frac{M+\sqrt{M^{2}-c(t)}}{\sqrt{c(t)}}\right)\right] .
$$

Now one should specify the time dependence of $c(t)$. Using equation (5), since there exists several possibilities for $a(t)$ and $c(t)=M_{P}^{2}(t)=a^{n}(t)$, we can consider de Sitter Universe as an example,

$$
c(t)=\left[a_{0} \cosh \left(\frac{t}{a_{0}}\right)\right]^{n} .
$$


Now, equations (12)-(15) for de Sitter Universe become respectively,

$$
\begin{gathered}
T_{H}^{(V S L)}(t)=\frac{\cosh ^{3 n}(t)}{8 \pi M}, \\
S_{B}^{(V S L)}(t)=\frac{4 \pi M^{2}}{\cosh ^{n}(t)}, \\
T_{G U P}^{(V S L)}(t)=\frac{M \cosh ^{2 n}(t)}{4 \pi}\left[1-\sqrt{1-\frac{\cosh ^{n}(t)}{M^{2}}}\right],
\end{gathered}
$$

and

$S_{G U P}^{(V S L)}(t)=2 \pi\left[\frac{M^{2}}{\cosh ^{n}(t)}\left(1-\frac{\cosh ^{n}(t)}{M^{2}}+\sqrt{1-\frac{\cosh ^{n}(t)}{M^{2}}}\right)-\ln \left(\frac{M+\sqrt{M^{2}-\cosh ^{n}(t)}}{\sqrt{\cosh ^{n}(t)}}\right)\right]$.

Where we have set $a_{0}=1$. The results of numerical calculations are shown in figures. In these figures we have considered $n=-1$ and the results are shown for de Sitter Universe. Note that for different $n$, the overall behavior of the solutions do not change considerably and other model Universes give similar results.

\section{Conclusions and Discussion}

Based on our model and numerical calculations, the following results are obtained

1- When one considers the time variation of speed of light alone, total evaporation of black hole is possible in principle. Thus in the framework of VSL micro black hole can evaporate completely. This is in agreement with the Hawking-Bekenstein and in contrast with the results of GUP.

2- Application of generalized uncertainty principle to black holes thermodynamics strongly suggests the possible existence of black holes remnant (figure 1 and 2).

3- When one considers thermodynamics of black holes in the framework of both GUP and VSL, some novel results are obtained. The figure for temperature of black hole versus the mass and time in a combination of GUP and VSL (figure 5) shows that where the mass of black hole is zero, its temperature is zero also. This is in contrast 
to Hawking result and seems completely reasonable since in the absence of matter there is no meaning for temperature. When the mass increases, the temperature is increases until the mass becomes equal to the Planck mass. After that, increasing of mass is corresponding to decreasing of temperature in complete agreement with the results of GUP (Adler et al [29]). But the situation for mass less than Planck mass is completely different from GUP results.

4- The figure for entropy of black hole versus the mass and time in a combination of GUP and VSL (figure 6) shows that when one approaches the Planck mass, entropy do not vanishes. This is physically reasonable but rules out the result of Adler et $a l$ since they have zero entropy for remnants. Increasing the time will increase the entropy which is natural. The possibility of having black hole remnant at Planck mass is evident from this figure. In our model there is a remnant entropy for black remnant. This can be at least related to background spacetime metric fluctuation.

5- Adler and his coworkers have constructed their formulation based on analogy between hydrogen atom and black holes. They have argued that since uncertainty principle prevents the hydrogen atom from total collapse, generalized uncertainty principle may prevent black holes total evaporation in the same manner. In our opinion, the basic mistake of Adler et al is that they have not considered hydrogen atom in GUP. Our calculation shows that in GUP hydrogen atom is not stable. Since,

$$
\Delta r \Delta p \geq \frac{\hbar}{2}\left(1+\beta(\Delta p)^{2}\right)
$$

Suppose that $\Delta p \sim p$ and $\Delta r \sim r$, then one finds

$$
p r=\frac{\hbar}{2}\left(1+\beta p^{2}\right) \Rightarrow \hbar \beta p^{2}-2 p r+\hbar=0 .
$$

So one obtains,

$$
p=\frac{r \pm \sqrt{r^{2}-\beta \hbar^{2}}}{\beta \hbar} .
$$

As has been argued we should consider the minus sign in (23). Since

$$
E=\frac{p^{2}}{2 m}-\frac{e^{2}}{r}
$$

one find,

$$
E=\frac{1}{2 m}\left(\frac{r-\sqrt{r^{2}-\beta \hbar^{2}}}{\beta \hbar}\right)^{2}-\frac{e^{2}}{r} .
$$


Now we set, $\frac{d E}{d r}=0$ and find $r_{\min }=\hbar \sqrt{\frac{\beta}{2}}$. The extremum value of energy becomes,

$$
E_{\min }=-\left[\left(\frac{e^{2}}{\hbar} \sqrt{\frac{2}{\beta}}\right)+i \frac{1}{2 m \beta}\right]
$$

where its real part is,

$$
E_{\text {min }}^{\text {real }}=-\left(\frac{e^{2}}{\hbar} \sqrt{\frac{2}{\beta}}\right) .
$$

Since $r_{\min }$ is very small length, the radius of stability for hydrogen atom is very small. Therefore in GUP scale, the hydrogen atom is not stable and will collapse completely. If this is the case, one can not construct analogy between hydrogen atom in Heisenberg uncertainty principle viewpoint and black hole in GUP viewpoint. If we consider hydrogen atom in GUP, as we have shown, this atom will collapse totally. But in the framework of GUP black holes evaporate until they reach Planck mass. The issue of stability for remnant can be considered in the framework of symmetry principle in the system. In this regard supersymmetry, in particular supergravity, stands a very good framework of providing such black hole remnants [30].

Note that our arguments for the existence of black hole remnants based on GUP is heuristic. The search for its deeper theoretical foundation is currently underway. As interactions with black hole remnants are purely gravitational, the cross section is extremely small, and direct observation of these remnants seems unlikely. One possible indirect signature may be associated with the cosmic gravitational wave background. Unlike photons, the gravitons radiated during evaporation would be instantly frozen. Since, according to our notion, the black hole evaporation would terminate when it reduces to a remnants, the graviton spectrum should have a cutoff at Planck mass. Such a cutoff would have by now been red-shifted to $\sim 10^{14} \mathrm{GeV}$. Another possible gravitational wave-related signature may be the gravitational wave released during the gravitational collapse. The frequencies of such gravitational waves would by now be in the range of $\sim 10^{7}-10^{8} \mathrm{~Hz}$. It would be interesting to investigate whether these signals are in principle observable. Another possible signature may be some imprints on the CMB fluctuations due to the thermodynamics of black hole remnants-CMB interactions. Possible production of such remnants in LHC (Large Hadron Collider) and also in cosmic ray showers are under investigation. If we consider hybrid inflation as our primary cosmological model, there will be some observational constraints on hybrid inflation parameters. For example 
a simple calculation based on hybrid inflation suggests that the time it took for black holes to reduce to remnants is about $10^{-10} \mathrm{Sec}$. Thus primordial black holes have been produced before baryogenesis and subsequent epochs in the standard cosmology [30].

\section{Acknowledgment}

This Work has been supported partially by Research Institute for Astronomy and Astrophysics of Maragha, Iran. Also we would like to appreciate an unknown referee of MPLA for his (her) valuable comments on original version of the paper.

\section{References}

[1] P. Gondolo, Lectures delivered at the NATO Advanced Study Institute "Frontiers of the Universe", 8-20 Sept 2003, Cargese, France; astro-ph/0403064.

[2] Ya. B. Zeldovich and I. D. Novikov, Sov. Astron. 10 (1966) 602.

[3] S. W. Hawking, Mon. Not. R. Astron. Soc. 152 (1971) 75.

[4] E. J. Copeland et al, Phys. Rev. D 49 (1994) 6410.

[5] D. H. Lyth and A. Riotto, Phys. Rep. 314 (1999) 1; A. Linde, Phys. Rep. 575 (2000) 333.

[6] G. Veneziano, Europhys. Lett. 2 (1986) 199; Proc. of Texas Superstring Workshop (1989).

[7] D. Amati, M. Ciafaloni and G. Veneziano, Phys. Lett. B 216 (1989) 41.

[8] D. Amati, M. Ciafaloni and G. Veneziano, Phys. Lett. B 197 (1987) 81; Int. J. Mod. Phys. A 3 (1988) 1615; Nucl. Phys. B 347 (1990) 530.

[9] D. J. Gross and P. F. Mende, Phys. Lett. B 197 (1987) 129; Nucl. Phys. B 303 (1988) 407.

[10] K. Konishi, G. Paffuti and P. Provero, Phys. Lett. B 234 (1990) 276; R. Guida, K. Konishi and P. Provero, Mod. Phys. Lett. A 6 (1991) 1487. 
[11] M. Kato, Phys. Lett. B 245 (1990) 43.

[12] L. J. Garay, Int. J. Mod. Phys. A 10 (1995) 145.

[13] S. Capozziello, G. Lambiase and G. Scarpetta, Int. J. Theor. Phys. 39 (2000) 15.

[14] M. Maggiore, Phys. Lett. B 304 (1993) 65.

[15] C. Castro, Found. Phys. Lett. 10 (1997) 273.

[16] A. Camacho, Gen. Rel. Grav. 34 (2002) 1839.

[17] M. Maggiore, Phys. Rev. D 49 (1994) 5182.

[18] M. Maggiore, Phys. Lett. B 319 (1993) 83.

[19] S. Kalyana Rama, Phys. Lett. B 519 (2001) 103.

[20] A. Camacho, Gen. Rel. Grav. 35 (2003) 1153.

[21] F. Scardigli and R. Casadio, Class. Quant. Grav. 20 (2003) 3915.

[22] S. Hossenfelder, Mod. Phys. Lett. A 19 (2004) 2727.

[23] S. Hossenfelder, Phys. Rev. D 70 (2004) 105003.

[24] S. Hossenfelder, Mod. Phys. Lett. A 19 (2004) 2727.

[25] A. J. M. Medved and E. C. Vagenas, Phys. Rev. D 70 (2004) 124021.

[26] A. J. M. Medved, Class. Quant. Grav. 22 (2005) 133.

[27] B. Bolen and M. Cavaglia, Gen. Rel. Grav. 37 (2005) 1255.

[28] M. R. Setare, Phys. Rev. D 70 (2004) 087501.

[29] P. Chen and R. J. Adler, Nucl. Phys. B (Proc.Suppl.) 124 (2003) 103; R. J. Adler, P. Chen and D. I. Santiago, Gen. Rel. Grav. 33 (2001) 2101.

[30] P. Chen, New Astron. Rev. 49 (2005) 233.

[31] A. Albrecht and J. Magueijo, Phys. Rev. D 59 (1999) 043516.

[32] J. Magueijo, Phys. Rev. D 62 (2000) 103521. 
[33] J. D. Barrow and J. Magueijo, Phys. Lett. B 443 (1998) 104; Phys. Lett. B 447 (1999) 246; Class. Quant. Grav. 16 (1999) 1435.

[34] J. Magueijo, Phys. Rev. D 63 (2001) 043502.

[35] J. D. Barrow, Phys. Rev. D 59 (1999) 043515.

[36] A. Kempf et al., Phys. Rev. D 52 (1995) 1108. 


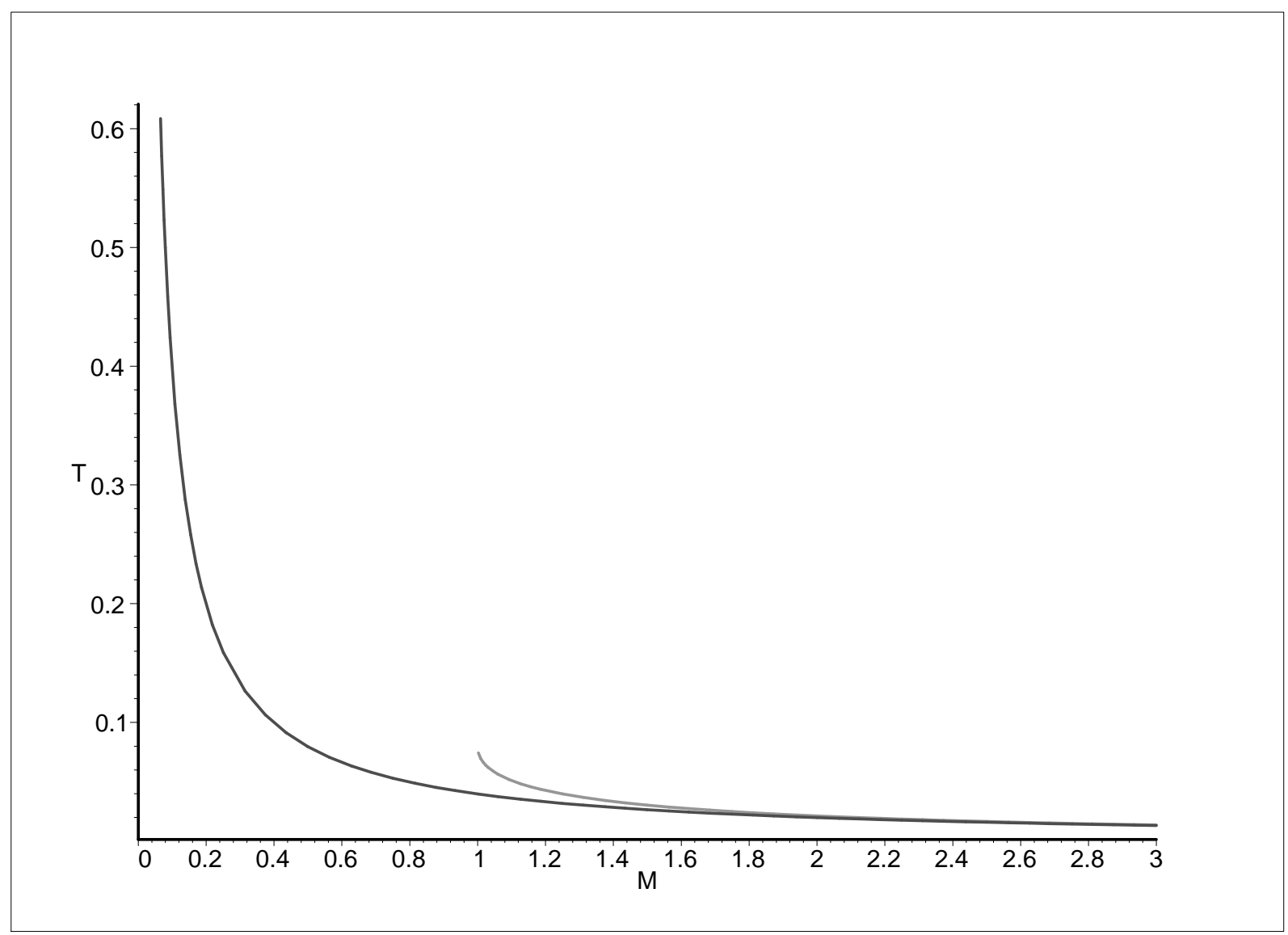

Figure 1: Temperature of a Black Hole Versus the Mass. Mass is in units of the Planck mass and temperature is in units of the Planck energy. The lower curve is the Hawking result, and the upper curve is the result of GUP. 


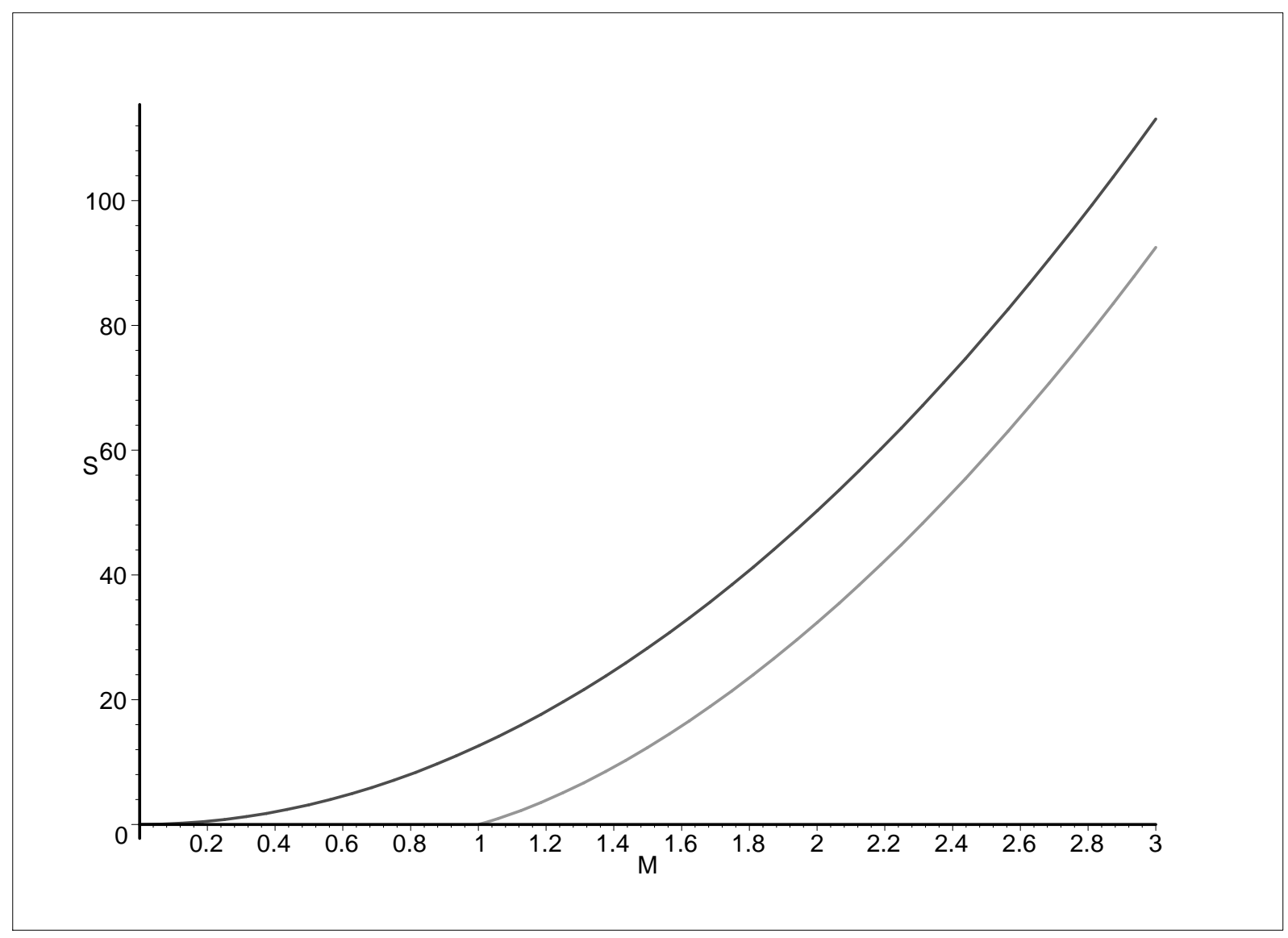

Figure 2: Entropy of a Black Hole Versus the Mass. Entropy is dimensionless and mass is in units of the Planck mass. The upper curve is the Hawking result, and the lower curve is the result of GUP. 


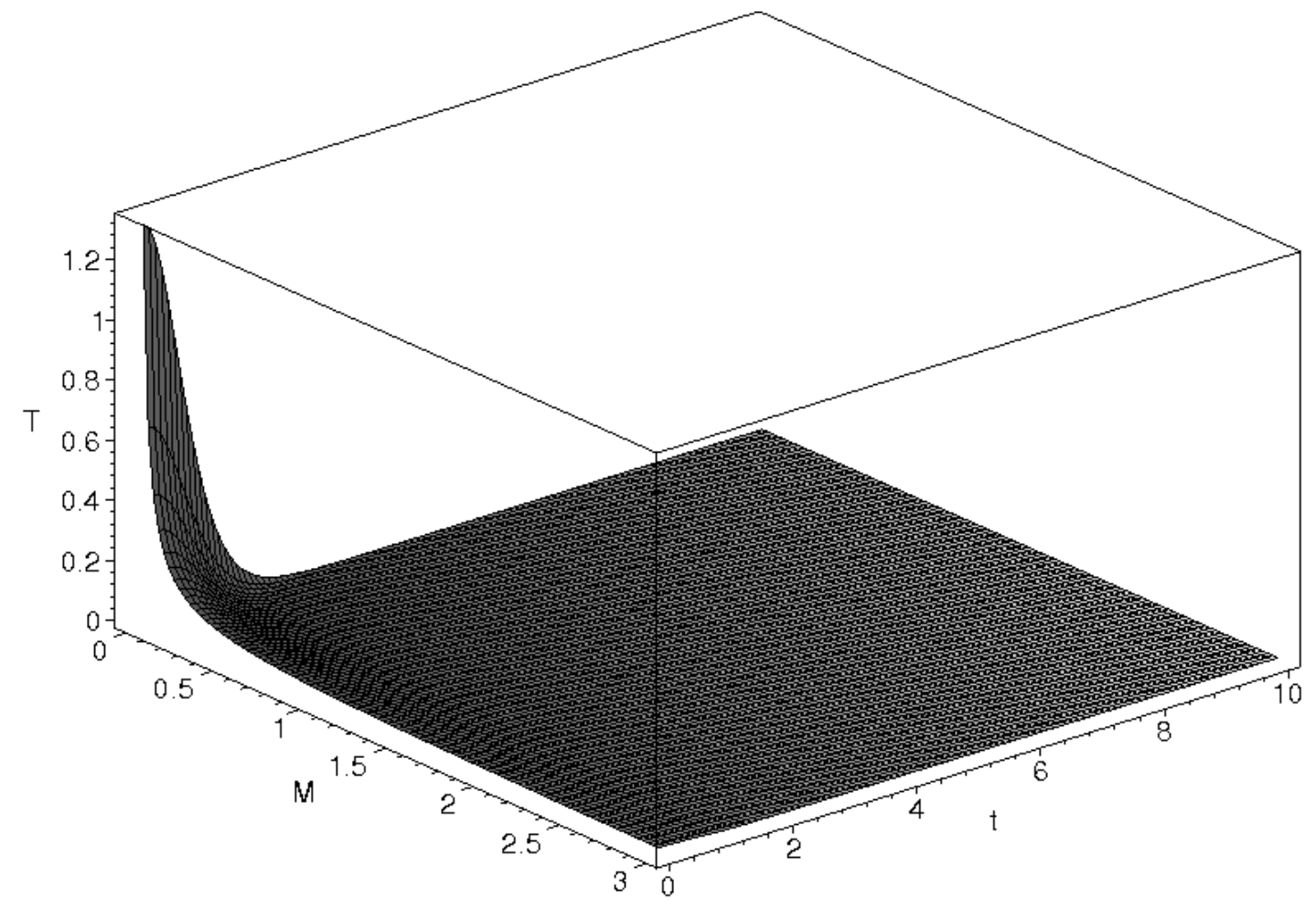

Figure 3: Temperature of Black Hole Versus the Mass and Time in VSL. The units are as previous and the result is shown for De Sitter model. As figure shows, the result of Hawking is recovered. Decreasing of temperature with increasing of time is natural. 


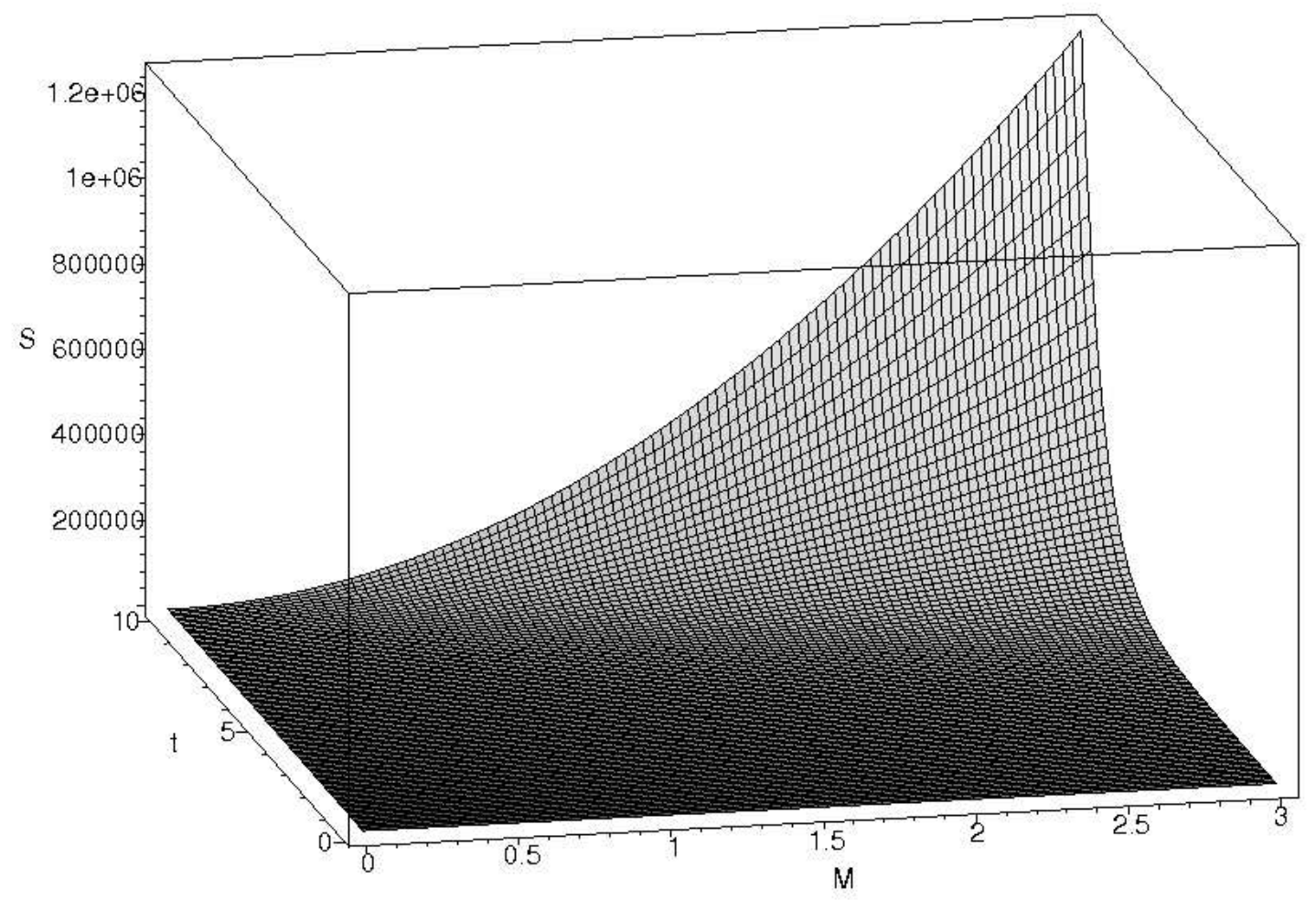

Figure 4: Entropy of Black Hole Versus the Mass and Time in VSL. The units are as previous and the result is shown for De Sitter model. As figure shows, the result of Hawking is recovered. Increasing of Entropy with increasing of time is natural. 


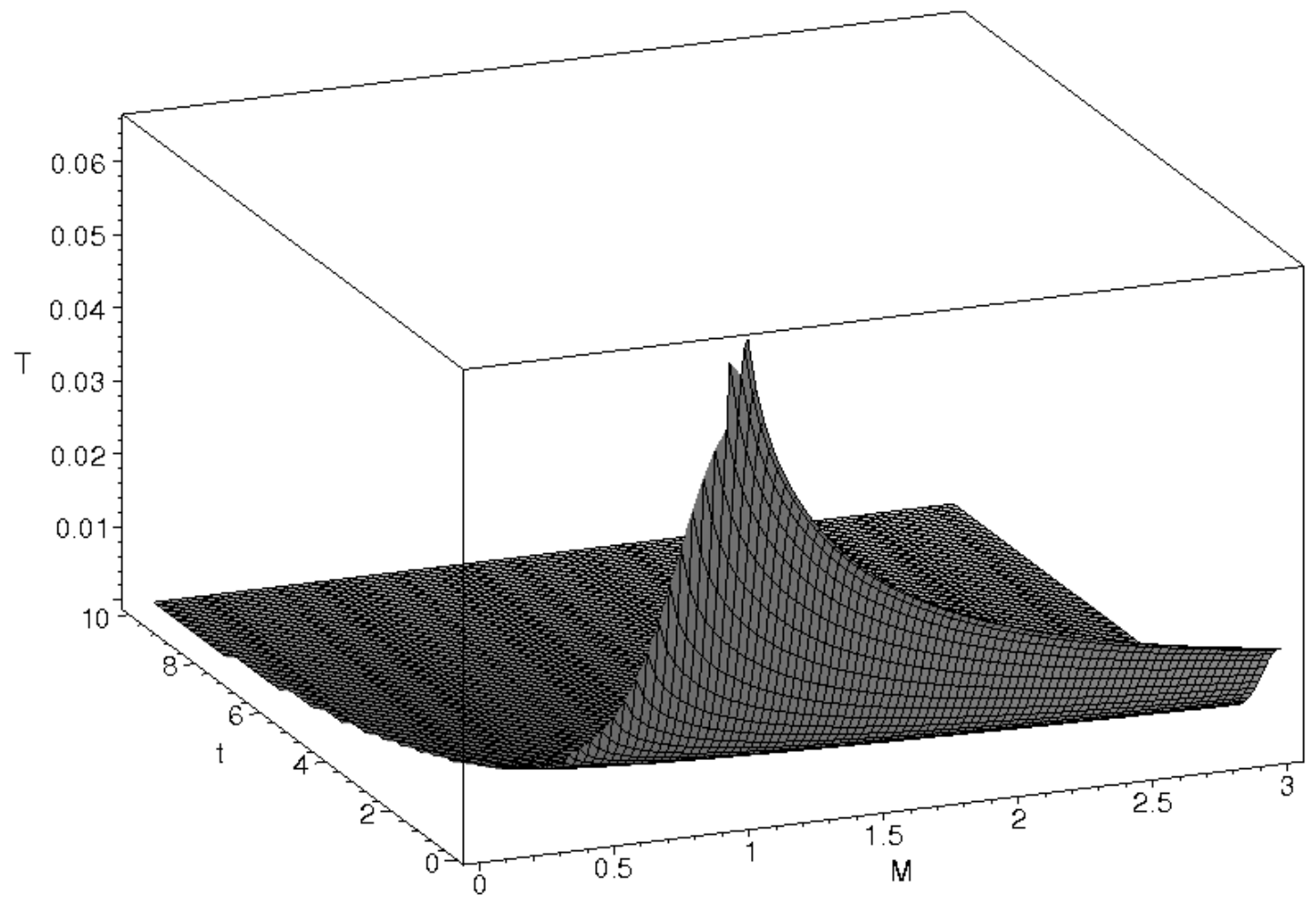

Figure 5: Temperature of Black Hole Versus the Mass and Time in a combination of GUP and VSL. The units are as previous and the result is shown for De Sitter model. As figure shows, where the mass is zero the temperature is zero also. This is in contrast to Hawking result. When the mass increases, the temperature increases until the mass becomes equal to the Planck mass. After that, increasing of mass is corresponding to decreasing of temperature. This is a novel result of GUP+VSL Scenario. 


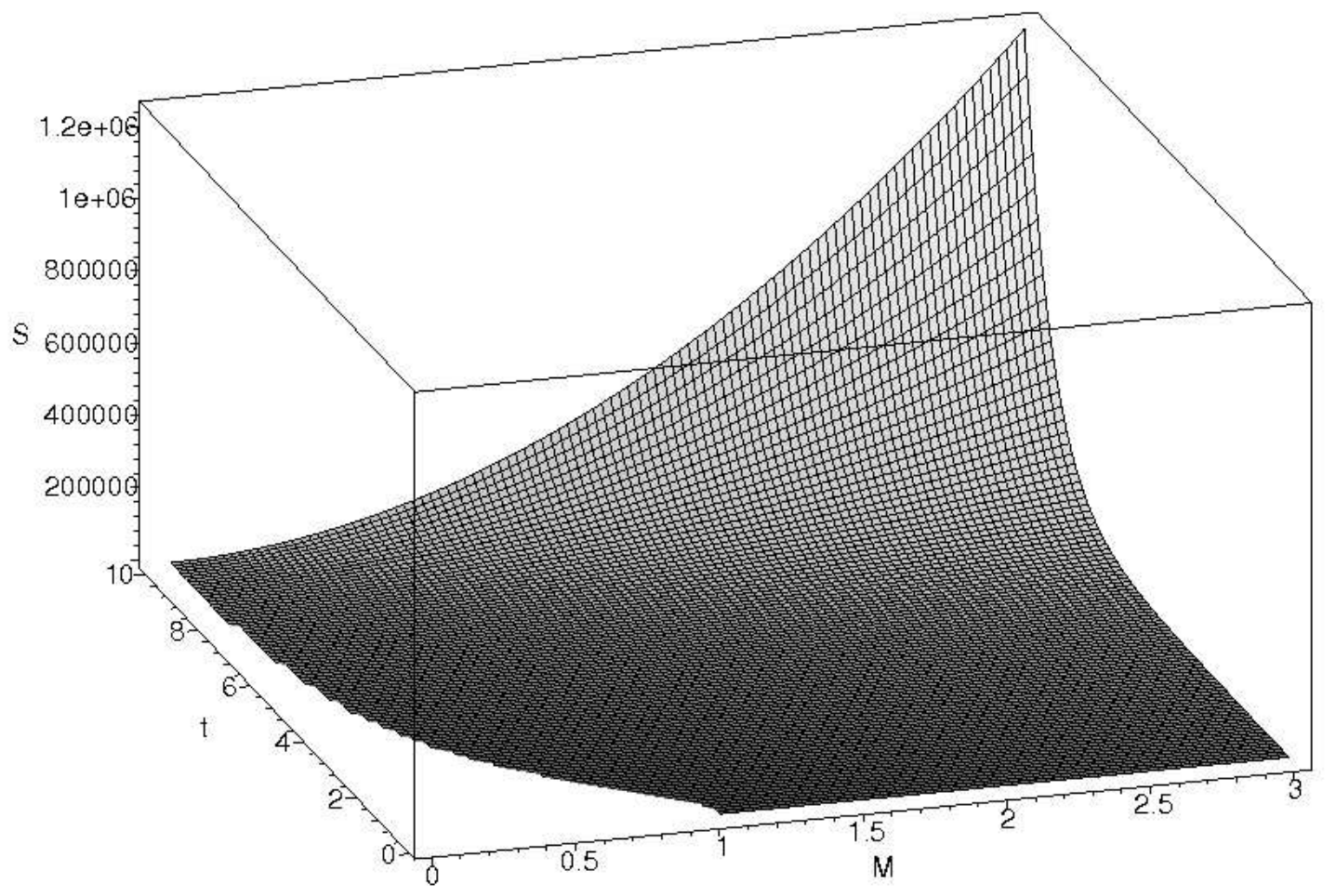

Figure 6: Entropy of Black Hole Versus the Mass and Time in a Combination of GUP and VSL. The units are as previous and the result is shown for De Sitter model. As figure shows, When one approaches the Planck mass, entropy do not vanishes. Increasing entropy with time is natural. This figure shows the possibility of having black holes relics. 